\title{
Spectrometry of pulsed photon radiation
}

\author{
Rolf Behrens $^{1}$, Hayo Zutz ${ }^{1}$, Julian Busse ${ }^{1}$ \\ ${ }^{1}$ Physikalisch-Technische Bundesanstalt (PTB), Bundesallee 100, 38116 Braunschweig \\ Rolf.Behrens@PTB.de
}

\section{Summary:}

The energy distribution (spectrum) of pulsed photon radiation can hardly be measured using active devices. Therefore, a thermoluminescence detector (TLD)-based few-channel spectrometer is used in combination with a Bayesian data analysis.

Keywords: Spectrometry, Pulsed photon radiation, Bayesian analysis, Uncertainty

\section{Background, Motivation and Objective}

Pulsed photon radiation arises in more and more situations, e.g. from small pulsed X-ray tubes for material (like weld seam) testing or linear accelerators in tumor therapy. In this work, spectra at such radiation fields are measured.

\section{Measurements and Data Evaluation}

For the measurements, a TLD-based few-channel spectrometer (FCS) is used (see Fig. 1) [1],[2]. The photons' penetration depth in the spectrometer depends on the energy so that the energy-resolved and absolute spectrum of the radiation, including the uncertainties of the spectrum, can be determined from the dose values in the TLD layers using Bayesian data evaluation (deconvolution).

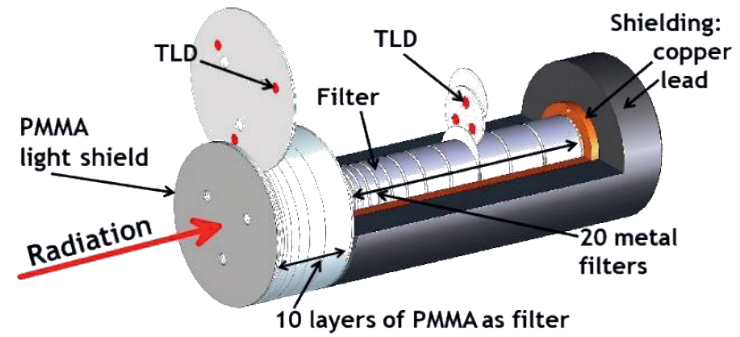

Fig. 1. Sketch of the TLD-based spectrometer. Basic principle: The deeper the radiation penetrates the spectrometer, the higher its energy.

The experimental setups are shown in Fig. 2: At the top, the spectrum of an industrial type open beam X-ray generator, XRS4 with a tube voltage of $370 \mathrm{kV}$, is measured. In the same way the spectrum of the XR200 with a tube voltage of $150 \mathrm{kV}$ is measured. At the bottom, the spectrum of a medical accelerator at nominal $25 \mathrm{MV}$ (which is actually $20 \mathrm{MV}$ real) high voltage behind a shielding wall, is measured. The latter field is considered as reference field of pulsed high energy photons for dosemeter testing.

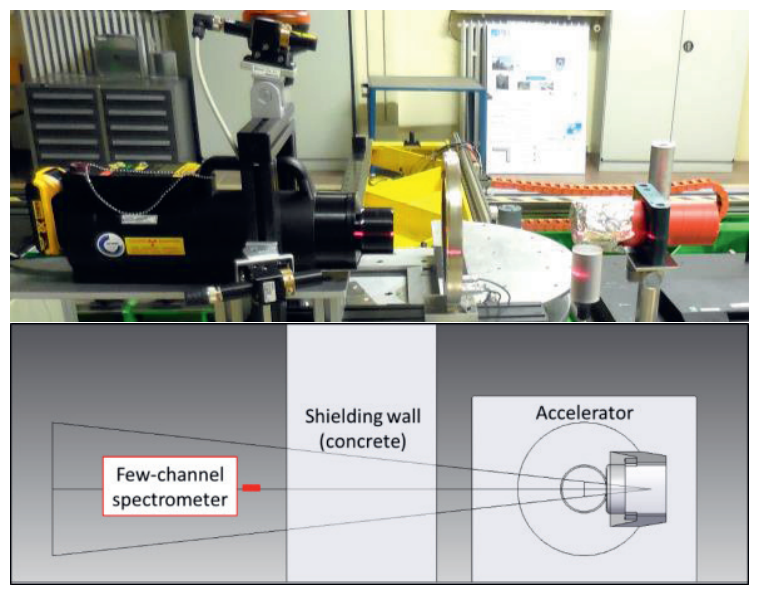

Fig. 2. Experimental setups. Top: industrial type open beam X-ray generator, XRS4, left, and FCS (red), right. Bottom: sketch of the medical accelerator, right, $2 \mathrm{~m}$ shielding wall, middle, FCS (red), left.

The TLDs are calibrated absolutely in terms of air kerma at PTB's corresponding reference field (Cs-137 radiation).

The Bayesian data evaluation is performed using the WinBUGS software [3] which, besides the absolute photon spectrum and total doses, also supplies the corresponding uncertainties and coverage intervals.

The following prior information for the photon spectra is included in the data evaluation: i) a smooth rise with increasing energy, ii) an exponential decrease at higher energies and iii) a peak in the spectrum at the energy of the characteristic fluorescence radiation of the anode material (for the XRS4 and XR200). This prior information is used due to the well-known form of bremsstrahlung spectra. Further details, including the validation of the method, (irradiation in known photon fields and subsequent data evaluation with the same prior information) are given in the literature [2],[5],[6]. 


\section{Results}

Fig. 3 shows the absolute photon fluence per pulse from the XRS4 and XR200 normalized to a distance of $1 \mathrm{~m}$, top, and the absolute photon fluence per absorbed dose to water at the ISO center of the medical accelerator (at $1 \mathrm{~m}$ distance) at a total distance of $5.5 \mathrm{~m}$ from the accelerator. The latter one is measured at two different cross-sectional beam areas (at $1 \mathrm{~m}$ from the accelerator): $40 \times 40 \mathrm{~cm}^{2}$ and $10 \times 10 \mathrm{~cm}^{2}$. In both graphs, also the spectrum used as starting point for the Bayesian data evaluation is given. The fluence spectra are converted to ambient dose equivalent, $H^{*}(10)$, using the corresponding conversion coefficients [4]. The resulting doses are given in the legends together with the spectra's mean energies, $\bar{E}$, and their conversion coefficient from air kerma, $K_{\mathrm{a}}$, to $H^{\star}(10): h^{*} K(10)$.

The top of Fig. 3 reveals that the data evaluation clearly identifies the end point energies of the X-ray tubes. Furthermore, for the XRS4 compared to the XR200, the mean energy is approximately 1.5 times, the dose per pulse 2 times and the conversion coefficient rather similar. The differences are as expected due to the larger tube voltage of the XRS4.

The bottom of Fig. 3 shows that the data evaluation also identifies the end point energy of the accelerator. Furthermore, for the $40 \times 40 \mathrm{~cm}^{2}$ field compared to the $10 \times 10 \mathrm{~cm}^{2}$ field, the mean energy is approximately $30 \%$ smaller, the dose per reference dose almost 2 times larger and the conversion coefficient nearly the same. The differences are as expected due to the larger beam area of the $40 \times 40 \mathrm{~cm}^{2}$ field resulting in a larger contribution of stray radiation at the spectrometer's position from within the shielding wall. As the photons lose energy during their scattering in the wall, the mean energy is smaller.

\section{Conclusions}

The measurements clearly show that the few-channel spectrometer in combination with the Bayesian data evaluation can be used in different areas of application to reliably measure the spectrum of pulsed photon radiation, including uncertainties, coverage intervals and doses.

\section{Funding}

This work is supported by the Bundesamt für Strahlenschutz (BfS) with project no. 3619S2236 / FV-63014 „Aufbau und Charakterisierung eines Referenzfeldes zur Sicherstellung des Strahlenschutzes an Beschleunigeranlagen in Medizin und Forschung und zur Prüfung und Kalibrierung entsprechender Messgeräte“.
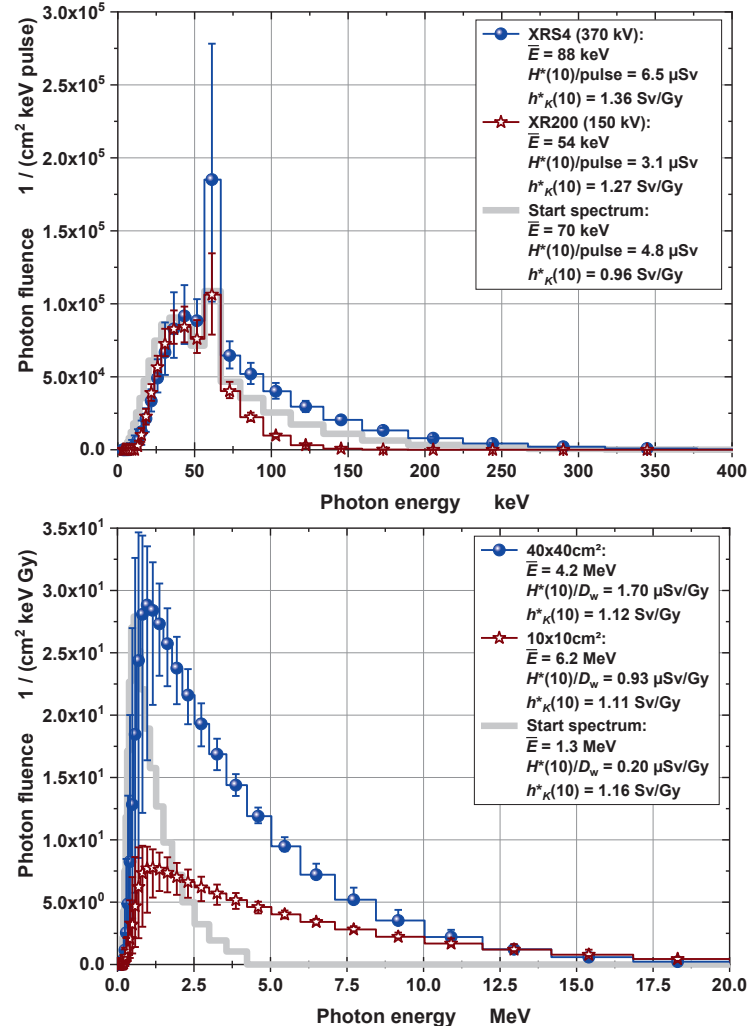

Fig. 3. Photon fluence spectra together with their $95 \%$ coverage intervals. Top: $X$-ray generator, XRS4 (370 kV tube voltage) and XR200 (150 kV tube voltage); bottom: shielded, pulsed high energy photon field from a medical accelerator at nominal $25 \mathrm{MV}$ high voltage.

\section{References}

[1] R. Behrens and P. Ambrosi, A TLD-based Fewchannel Spectrometer for Mixed Photon, Electron, and Ion Fields with High Fluence Rates, Radiat. Prot. Dosim. 101, 73 (2002) doi: 10.1093/oxfordjournals.rpd.a006062

[2] R. Behrens, A spectrometer for pulsed and continuous photon radiation, J. Instrum. 4, P03027 (2009) doi: 10.1088/17480221/4/03/P03027

[3] D.J. Lunn, A. Thomas, N. Best, D. Spiegelhalter, WinBUGS - a Bayesian modelling framework: Concepts, structure, and extensibility, Statistics and Computing 10, 325 (2010) doi: 10.1023/A:1008929526011

[4] International Commission on Radiation Units and Measurements (ICRU), Conversion Coefficients for use in Radiological Protection against External Radiation. ICRU Report 57 (1998) doi: 10.1093/jicru/os29.2.Report57

[5] R. Behrens, B. Pullner, M. Reginatto, X-Ray Emission from Materials Processing Lasers, Radiat. Prot. Dosim. 183, 361-374 (2019); doi: $10.1093 / \mathrm{rpd} / \mathrm{ncy} 126$

[6] R. Behrens, M. Reginatto, Bayesian Spectrum Deconvolution Including Uncertainties and Model Selection: Application to X-Ray Emission Data Using WinBUGS, Radiat. Prot. Dosim. 185, 157167 (2019); doi: 10.1093/rpd/ncy286 